\title{
A comparative analysis of the electron energy distribution function obtained by regularization methods and by a least-squares fitting.
}

\author{
C. Gutiérrez-Tapia and H. Flores-LLamas \\ Departamento de Física, Instituto Nacional de Investigaciones Nucleares \\ A. Postal 18-1027, 11801 México D. F., MEXICO \\ E-mail: cgt@nuclear.inin.mx
}

\begin{abstract}
To establish the electron energy distribution function (EEDF), the second derivative of a Langmuir probe current-voltage (I-V) characteristic is numerically integrated using the Tikhonov singular value decomposition regularized method. A comparison of the numerically intagrated EDDF and by a least-squares fitting is discussed. The used I-V characteristic is measured in an ECR plasma source using a cylindrical probe and the plasma parameters are determined by the Laframboise theory. This technique allows a rapid analysis of plasma parameters at any gas pressure. The obtained EEDF, for the case of the ECR plasma source, shows the existence of two groups of electrons with different temperatures. This result is associated with the collisional mechanism heating taking place in ECR plasma sources, where low pressure plasma is sustained by electron impact ionization of the ground state molecules or atoms by energetic electrons arising in the resonance zone.
\end{abstract}

PACS numbers: 52.70.Ds, 52.50.Sw, 52.80.Pi 


\section{Introduction.}

The Langmuir probe is one of the simplest diagnostics tools of the study of ionized gases. The probe usually consists of a small sphere or a circular cylinder which is placed in the plasma at the point of interest. Some external circuitry is provided so that the electric potential of the probe can be varied. A plot of the total current flowing between the probe and the plasma versus the probe potential is called the current-voltage (IV) characteristic. Langmuir probes are highly effective in determining the electron energy distribution function (EEDF), allowing both spatial and temporal resolution. The EEDF is given by the second derivative of the probe I-V characteristic.

In recent years, several groups have successfully used the numerical differentiation of digital probe trace data. [1, 2, 3. Yet, numerically, the second derivative is a hard task and is classified as an ill-posed problem. Ill-posed problems do not have the properties of existence, uniqueness and stability [4]. One of the alternatives to solve this problem is the introduction of the concept of conditional well-posed problems [4]. On the other hand, the problem of integration of the I-V characteristic has evolved along with the solution of the Fredholm integral equation of first class, which is obtained from the integration of a second order differential equation. One of the most stable methods to solve the Fredholm integral equation are the regularization methods.

In order to characterize the processes occurring in plasmas it is advisable to obtain the electron energy distribution function with a high degree of accuracy. In particular, there has been much interest in the electron cyclotron resonance (ECR) plasma sources motivated by their applications in ionized physical vapor deposition techniques (I-PVD) [5].

In this paper, the second derivative of a Langmuir probe current-voltage (I-V) characteristic is numerically integrated using the Tikhonov singular value decomposition (SVD) regularized method in order to establish the EEDF as the most stable method. The existence of two groups of electrons with different temperatures, where low pressure plasma is sustained by electron impact ionization of the ground state molecules or atoms by energetic electrons arising in the resonance zone is shown. Also, it is important to notice that the rate of convergence of the Tikhonov method is much faster than that achieved with the least-squares with an orthogonal decomposition (LSQR) and truncated singular value decomposition (TSDV) methods. A comparison between the EEDF obtained by the Tikhonov regularization method and the one resulting from a least-squares fitting is discussed.

\section{Basic equations.}

The electric potential profile near the probe can influence its current collection by setting up "barriers of effective potential" around the probe, thereby preventing some particles from reaching it. This profile can be strongly influenced by the space charge of the particles themselves. However, when $r_{p} / \lambda_{D}$ is small enough (the sheath around the probe 
is comparatively thick), this barriers disappears, and the current collection is maximized. The currents collected under these conditions then become the orbit-limited-currents. This assumption is backed by the orbital motion limit (OML) theory [6] which implies a thick, collisionless sheath $\left(\lambda_{e} \gg r_{p}\right)$. Thus, the calculation of Laframboise [6], based on the more complete theory of Berstein and Rabinowitz [7, showed that the OML limit is achived for $\lambda_{D} \approx r_{p}$. Here $r_{p}$ is the probe radius (spherical or cylindrical), $\lambda_{D}$ is the Debye radius, and $\lambda_{e}$ is the electron mean free path, respectively.

When we assume the presence of a stopping field $\left(\lambda_{e}>r_{p}>\lambda_{D}\right)$, as demonstrated by Druyvesteyn in [8, 9, the second derivative of the current $I$ with respect to the voltage $V$ of the probe is proportional to the electron energy distribution function as follows

$$
F(\varepsilon)=n_{e} f(\varepsilon)=\frac{4}{e S} \sqrt{\frac{m V_{p}}{2 e}} \frac{d^{2} I}{d V_{p}^{2}},
$$

where the energy is $\varepsilon=e\left(V_{p}-V_{s}\right), V_{p}$ is the probe voltage, $V_{s}$ is the plasma potential, $I$ is the electron current to the probe, $S$ is the probe surface area, and $m, e$ are the electron mass and charge, respectively. The contribution of the ion current to the second derivative of the probe current is normally insignificant, and it is therefore not subtracted in these EEDF calculations.

The equation (11) is in the form

$$
\frac{d^{2} I}{d V_{p}^{2}}=F\left(V_{p}\right)
$$

where $F\left(V_{p}\right)$ is a function of the potential on the probe $V_{p}$ with the boundary conditions for the current given by

$$
\begin{aligned}
& \left.I\right|_{V_{p}=a}=I(a), \\
& \left.I\right|_{V_{p}=b}=I(b) .
\end{aligned}
$$

A double integration in $V_{p}$ of equation (2) results in [10]

$$
H\left(V_{p}\right)=\int_{a}^{b} K\left(V_{p}, V_{p}^{\prime}\right) F\left(V_{p}^{\prime}\right) d V_{p}^{\prime},
$$

where

$$
\begin{aligned}
& H\left(V_{p}\right)=I\left(V_{p}\right)-h\left(V_{p}\right), \\
& K\left(V_{p}, V_{p}^{\prime}\right)= \begin{cases}\frac{\left(a-V_{p}^{\prime}\right)\left(b-V_{p}\right)}{b-a}, & \text { if } V_{p}^{\prime} \leq V_{p} \\
\frac{\left(a-V_{p}\right)\left(b-V_{p}^{\prime}\right)}{b-a}, & \text { if } V_{p}^{\prime}>V_{p}\end{cases}
\end{aligned}
$$

and,

$$
h\left(V_{p}\right)=\frac{V_{p}}{b-a}[I(b)-I(a)]+\frac{1}{b-a}[b I(a)-a I(b)] .
$$

This integral equation is known as the Fredholm equation of first class and it can be solved numerically by regularization methods. The Galerkin discretization becomes the more efficient scheme for this class of problems [11, 12] . 
Table 1. Values of constants appearing in (20) and (21) obtained by a least-squares fitting.

\begin{tabular}{cccccc}
\hline$\gamma$ & $\beta$ & $V_{f}(\mathrm{~V})$ & $C_{0}$ & $A_{1}$ & $A_{2}$ \\
\hline 0.393 & $0.12 \times 10^{-3}$ & -13.49 & $0.161 \times 10^{-4}$ & $0.39 \times 10^{-6}$ & $0.131 \times 10^{-4}$ \\
$A_{3}$ & $V_{s}^{e}(\mathrm{~V})$ & $u_{1}$ & $u_{2}$ & $u_{3}$ & \\
$0.323 \times 10^{-7}$ & 25.3 & 0.096 & 0.188 & 0.0533 & \\
\hline
\end{tabular}

\section{EEDF by the Tikhonov regularization method.}

In order to solve the Fredholm's first class equation (44), the kernel $K$ can be expressed in the form of an expansion in terms of singular functions $u_{i}(x), v_{i}(x)$, and singular values $k_{i}$ :

$$
K(x, y)=\sum_{i=1}^{n} k_{i} u_{i}(x) v_{i}(y) .
$$

In the occurrence of symmetry, this reduces itself to an eigenfunction expansion. It is not necessary that the expansion should converge pointwise; all we require for an $L_{2}$ kernel is that

$$
\lim _{n \rightarrow \infty} \iint\left\{K(x, y)-\sum_{i=1}^{n} k_{i} u_{i}(x) v_{i}(y)\right\}^{2} d x d y=0,
$$

for which it is necessary and sufficient that the series $\sum_{i} k_{i}^{2}$ converge.

When we have an ill-conditioned matrix $K$, the $k_{i}$ values usually becomes very small. In this case the solution of the system (44) proposed by Tikhonov consists in replacing the ill-posed problem with a stable minimization problem involving a small positive parameter $\alpha$ : instead of attempting to solve the equation (44) directly, we seek to minimize the quadratic functional [4]

$$
\|K f-\tilde{h}\|^{2}+\alpha\|L f\|^{2}, K \in \Re^{m \times n}, m>n,
$$

where $L$ is some linear operator and $\tilde{h}$ denotes $h+\delta h$. If $L$ is suitably chosen, then the second term has a smoothing or stabilizing effect on the solution. We may, for example, take $L f=f, f^{\prime}$, or $f^{\prime \prime}$; if the $k-t h$ derivative is selected, the process is termed $k-t h$ order regularization. In the case $L f=f$, the solution of the minimization problem (10) is then obtained as the solution of the linear equation

$$
\left(K^{T} K+\alpha I\right) f_{\alpha}=K^{T} \tilde{h} .
$$

The operator acting on $f_{\alpha}$ is clearly positive-definite when $\alpha>0$ and consequently it has a bounded inverse. Solving in terms of singular functions we obtain

$$
f_{\alpha}(y)=\sum_{i} \frac{k_{i} h_{i}}{k_{i}^{2}+\alpha} v_{i}(y) .
$$

Comparing this with the exact expansion we can see that the effect of regularization has been to insert a filter factor $k_{i} /\left(k_{i}^{2}+\alpha\right)$. This is close to unity so long as $k_{i}$ is large 
A comparative analysis of the electron energy distribution function

Table 2. Values of plasma parameters obtained by the Langmuir theory, the Tikhonov regularization method and by a least-squares fitting.

\begin{tabular}{lcccccc}
\hline & $\begin{array}{c}T_{e} \\
(\mathrm{eV})\end{array}$ & $\begin{array}{c}n_{e} \\
\left(\mathrm{~m}^{-3}\right)\end{array}$ & $\begin{array}{c}\left.F(\varepsilon)\right|_{\max } \\
\left(\mathrm{m}^{-3} \mathrm{eV}^{-1}\right)\end{array}$ & $\begin{array}{c}V_{s} \\
(\mathrm{~V})\end{array}$ & $\begin{array}{c}n_{e} \\
\left(\mathrm{~m}^{-3}\right) \\
{[\mathrm{eq.} \mathrm{(22)]}]}\end{array}$ & $\begin{array}{c}T_{\text {eff }} \\
(\mathrm{eV}) \\
{[\text { eq. [23)] }}\end{array}$ \\
\hline 1st Derivative & 6.88 & $1.39 \times 10^{16}$ & - & 22.08 & - & - \\
Regularization & 5.66 & $1.54 \times 10^{16}$ & $7.34 \times 10^{14}$ & - & - & - \\
LS fitting & 5.331 & $1.62 \times 10^{16}$ & $1.131 \times 10^{15}$ & 25.3 & $7.8 \times 10^{15}$ & 8.4 \\
\hline
\end{tabular}

compared with $\alpha$ but tends to zero as $k_{i} \rightarrow 0$, the rate of transition depending on $\alpha$. If we split $\tilde{h}$ into $h+\delta h$ the expression (11) becomes

$$
f_{\alpha}=\sum_{i} \frac{k_{i} h_{i}}{k_{i}^{2}+\alpha} v_{i}(y)+\frac{k_{i} \delta h_{i}}{k_{i}^{2}+\alpha} v_{i}(y) .
$$

As for the first term, it is advantageous to make $\alpha$ small in order to reduce the error due to regularization; by contrast, the second term, which only consists of error, is made small by taking $\alpha$ large. Thus, there is a conflict, and we would like to achieve the best compromise [1].

\section{Least-squares fitting.}

In the OML theory of ion collection, the ion current flowing to a negatively biased probe is independent of the shape of the plasma potential $V(r)$ as long as the current is limited only by the angular momentum of the orbiting ions [6]. This requires the arbitrary assumption of either a "sheath edge" $s$, beyond which the ion energy distribution is Maxwellian, or a $V(r)$ varying so slowly that no "absorption radius", inside of which all ions are drawn in, exists between the probe and infinity. This condition is never satisfied even at modest densities. For $s \rightarrow \infty$ and a Maxwellian ion distribution at temperature $T_{i}$, the OML current to a cylinder probe is given by

$$
\begin{aligned}
I & =S j_{r}\left[\frac{2}{\sqrt{\pi}} \chi^{1 / 2}+e^{\chi}\left(1-\operatorname{erf}\left(\chi^{1 / 2}\right)\right)\right] \\
& \stackrel{\chi \gg 1}{\longrightarrow} S j_{r} \frac{2}{\sqrt{\pi}} \sqrt{1+\chi},
\end{aligned}
$$

where $\chi \equiv-e V_{p} / k T i$ and $j_{r}$ is the random thermal ion current. As $T_{i} \rightarrow 0$, the $T_{i}$ dependencies of $\chi$ and $j_{r}$ vanish, and a finite limiting value of the OML current is reached 13

$$
I \stackrel{T_{i} \rightarrow 0}{\longrightarrow} A_{p} n e \frac{\sqrt{2}}{\pi}\left(\frac{\left|e V_{p}\right|}{M}\right)^{1 / 2} .
$$

In the OML theory, the ion current of equation (14) can be represented as

$$
I\left(V_{p}\right)=-\beta\left(V_{p}-V_{f}\right)^{\gamma}+C_{0},
$$


where $\beta, \gamma, V_{f}$ and $C_{0}$ are constants to be fitted. In our case $V_{f}$ acquires the meaning of the float potential.

The three trial functions in the least-squares fitting of the I-V characteristic for the electronic component have the form

$$
F_{1}\left(V_{p}\right)=A_{1}\left(V_{p}-V_{s}\right) e^{-u_{1}\left(V_{p}-V_{s}\right)},
$$

and

$$
F_{i}\left(V_{p}\right)=A_{i}\left(V_{p}-V_{s}\right)^{2} e^{-\left[u_{i}\left(V_{p}-V_{s}\right)\right]^{2}}, \quad(i=2,3)
$$

where $A_{i}, u_{i},(i=2,3)$ and $V_{s}$ are constants that must be valued. Here $V_{s}$ adopts the character of the plasma potential.

Substituting equation (16) into an expression for the current to the probe, written in the form [13]

$$
I(V)=B_{1} \int_{x}^{\infty}\left(V_{p}-x\right) F\left(V_{p}\right) d V_{p},
$$

where $B_{1}=(4 / e S) \sqrt{1 / 2 m_{e}}, x=e V_{p}$ and in place of $F\left(V_{p}\right)$ we take any of functions (16)-(17), we obtain for the electronic component

$$
\frac{I_{1}\left(V_{p}\right)}{B_{1}}=\frac{A_{1}}{u_{1}}\left[2+u_{1}\left(V_{p}-V_{s}\right)\right] \exp \left[-u_{1}\left(V_{p}-V_{s}\right)\right],
$$

where the subindex 1 in $I$ refers to trial function (16). Analogously, from relations (17) we get

$$
\begin{aligned}
\frac{I_{i}\left(V_{p}\right)}{B_{1}}=\frac{A_{i}}{4 u_{i}^{2}}\left\{e \exp \left[-u_{i}^{2}\left(V_{p}-V_{s}\right)^{2}\right]\right. & \\
& \left.+u_{i} \sqrt{\pi}\left(V_{p}-V_{s}\right)\left(\operatorname{Erf}\left[u_{i}\left(V_{p}-V_{s}\right)\right]-1\right)\right\} \quad(i=2,3)
\end{aligned}
$$

\section{Analysis and discussion.}

With a view to obtaining accurate values of $n, T_{e}$ and $V_{s}$ (but not $T_{i}$ ) from the EEDF, we shall illustrate a procedure using data obtained in an ECR discharge with $P=10$ mTorr in argon gas, taken with an rf-compensated cylindrical probe with a radius 0.4 $\mathrm{mm}$ and $4 \mathrm{~mm}$ in length.

It is considered that the current is collected within the area at the tip of the probe expressed by $S=2 \pi R_{p} L+\pi R_{p}^{2}$, where $L$ is the probe length. The entire I-V curve in this example is shown in figure 1.

From the Langmuir theory, assuming a Maxwellian distribution for electrons [13], we obtain in the case of the $I-V$ curve of figure 1 that $T_{e}=6.88 \mathrm{eV}, V_{s}=22.078$ $\mathrm{V}$, and $n_{e}=1.39 \times 10^{16} \mathrm{~m}^{-3}$. Thus, the first derivative shown in figure 1 as well as experimental data are used. Here, it is important to notice that the extremum of the first derivative is not completely well defined, as observed in figure 1.

The EEDF is calculated following the procedure described in $\S 3$, by the integration of equation (4) with kernel (5), and using the Galerkin discretization method [14. 
Table 3. Values of plasma parameters obtained by the Langmuir theory, the Tikhonov regularization method and by a least-squares fitting for the energetic group of electrons.

\begin{tabular}{lccc}
\hline & $\begin{array}{c}T_{e} \\
(\mathrm{eV})\end{array}$ & $\begin{array}{c}n_{e}(\mathrm{~V}) \\
\left(\mathrm{m}^{-3}\right)\end{array}$ & $\begin{array}{c}\left.F(\varepsilon)\right|_{\max } \\
\left(\mathrm{m}^{-3} \mathrm{eV}^{-1}\right)\end{array}$ \\
\hline Regularization & 21.93 & $8.02 \times 10^{15}$ & $3.31 \times 10^{14}$ \\
LS fitting & 18.831 & $8.66 \times 10^{15}$ & $3.42 \times 10^{14}$ \\
\hline
\end{tabular}

In figure 3 the EEDF obtained by the Tikhonov technique of zeroth order is shown, along with the corresponding values of the regularization parameters $\alpha=(0.09,0.08$, $0.07,0.06,0.05,0.04,0.03,0.02$ ).

From these charts we gather that the most probable energy is $T_{e}=5.66 \mathrm{eV}$. With this electron temperature, it is easy to calculate the plasma electron density by the relation

$$
\left.I\left(V_{p}\right)\right|_{V_{p}=V_{s}}=e S n_{e}\left(\frac{T_{e}}{2 \pi m_{e}}\right)^{1 / 2},
$$

obtaining that $n_{e}=1.54 \times 10^{16} \mathrm{~m}^{-3}$. It is also important to notice that the maximum value of the EEDF by the Tikhonov regularization method takes the value of $\left.F(\varepsilon)\right|_{\max }=7.34 \times 10^{14} \mathrm{~m}^{-3} \mathrm{eV}^{-1}$. After an analysis of several calculations, we can say that the bumps observed in the EEDF are related with numerical effects and are originated by the irregularities of the I-V curve.

These results are validated in the following by a least squares fitting. As it has been described in $\S I V$ about the ionic part of the I-V characteristic, we fit the data to equation (16) determining the values for the constants $\gamma, \beta$ and $C_{0}$. These values are summarized in Table 1. This fitting is shown in figure 1. In figure 2, the measured values $I^{2}$ versus $V$ curves are plotted [13]. From these charts, we can observe that the obtained values for the constants show a good agreement with the data values.

Analogously, for the electronic component in the I-V curve, we now employ expressions (18) and (19) for a least squares fit to the data. The corresponding values of constants appearing in (18) and (19) are given in Table 11. Then, we can express the EEDF as a sum of functions (16)-(17). This distribution is plotted in figure 1. Introducing the corresponding dimensions, we obtain a comparison with the EEDF obtained by the Tikhonov regularization method shown in figure 3. For the energetic group of electrons we obtained that $T_{e}=18.831 \mathrm{eV}, n_{e}=8.66 E 15 \mathrm{~m}^{-3}$, and $\left.F(\varepsilon)\right|_{\max }=3.42 \mathrm{E} 13 \mathrm{~m}^{-3} \mathrm{eV}^{-1}$. From the least-squares distribution we obtain for the main group of electrons that the most probable energy is $T_{e}=5.331 \mathrm{eV}$. The maximum value found for the EEDF is $\left.F(\varepsilon)\right|_{\max }=1.131 \times 10^{15} \mathrm{~m}^{-3} \mathrm{eV}^{-1}$. Considering this value for the temperature $T_{e}$, we find by equation (21) the density $n_{e}=1.62 \times 10^{16} \mathrm{~m}^{-3}$. For the values computed after fitting, it is possible to accomplish the integration of the adjusted characteristic, to obtain [15, 16]

$$
n_{e}=\int_{0}^{\infty} F(\varepsilon) d \varepsilon=7.8 \times 10^{15} \mathrm{~m}^{-3},
$$


and

$$
T_{\text {eff }}=\frac{2}{3}\langle\varepsilon\rangle=2\left(3 n_{e}\right)^{-1} \int_{0}^{\infty} \varepsilon F(\varepsilon) d \varepsilon=8.40 \mathrm{eV}
$$

Here, the related problem of the indetermination of the zero position in the EEDF, as it is observed from figure 3, deserves some attention. This problem will always be present when no electronic saturation is reached. Several results obtained by applying the two methods are summarized in Table 2 and Table 3.

\section{Acknowledgments}

This work was partially supported by CONACyT, Mexico, under contract 33873-E.

\section{References}

[1] Hopkins M B and Graham W G 1987 J. Phys. D 20838

[2] Hopkins M B and Graham W G 1991 J. Appl. Phys. 693461

[3] Scalan J V and Hopkins M B 1992 J. Vac. Sci. Technol. A 10, 1207

[4] Tikhonov A N and Arsenin V Y 1977 Solutions of Ill-posed Problems (Washington D. C.: John Wiley \& Sons) p 45

[5] Camps E, Olea O, Gutiérrez-Tapia C and Villagrán M 1995 Rev. Sci. Instrum. 663219

[6] Leframboise G 1966 University of Toronto Institute of Aerospace Studies Report No. 100.

[7] Berstein I B and Rabinowitz I N 1959 Phys. Fluids 2212

[8] Druyvesteyn M J 1930 Zs. Phys. 64790

[9] Druyvesteyn M and Warmoltz N 1935 Phyl. Mag. 171

[10] Hildebrand F B 1992 Methods of Applied Mathematics (New York: Dover) p 222

[11] Delves L M and Walsh J 1974 Numerical Solution of Integral Equations (Oxford: Oxford Clarendon Press) p 175

[12] Hansen P 1994 Numerical Algorithms 61

[13] Chen F F 2001 Phys. Plasmas 83029

[14] Gutiérrez-Tapia C and Flores-Llamas H 2004 Phys. Plasmas 115102

[15] Klagge S and Lunk A 1991 J. Appl. Phys. 7088

[16] V. A. Godyak V A, Pijak R B, and Alexandrovich B M 1993 J. Appl. Phys. 733657 


\section{Figure captions}

Figure 1. Sample I-V curve to be analyzed obtained from an $0.4 \mathrm{~mm}$ diam, $4 \mathrm{~mm}$ long probe in an ECR discharge in 10 mTorr argon gas (solid). First derivative of the I-V curve (dotted), and saturation ion and electronic currents computed after fitting (dashed).

Figure 2. Square of saturation ion current vs probe voltage as measured (solid) and as computed after fitting (dashed).

Figure 3. EEDF resulting from the least squares fitting (solid) and by the Tikhonov regularization method (dotted). Here are shone the locations of the main and energetic groups of electrons respecting the energy. 


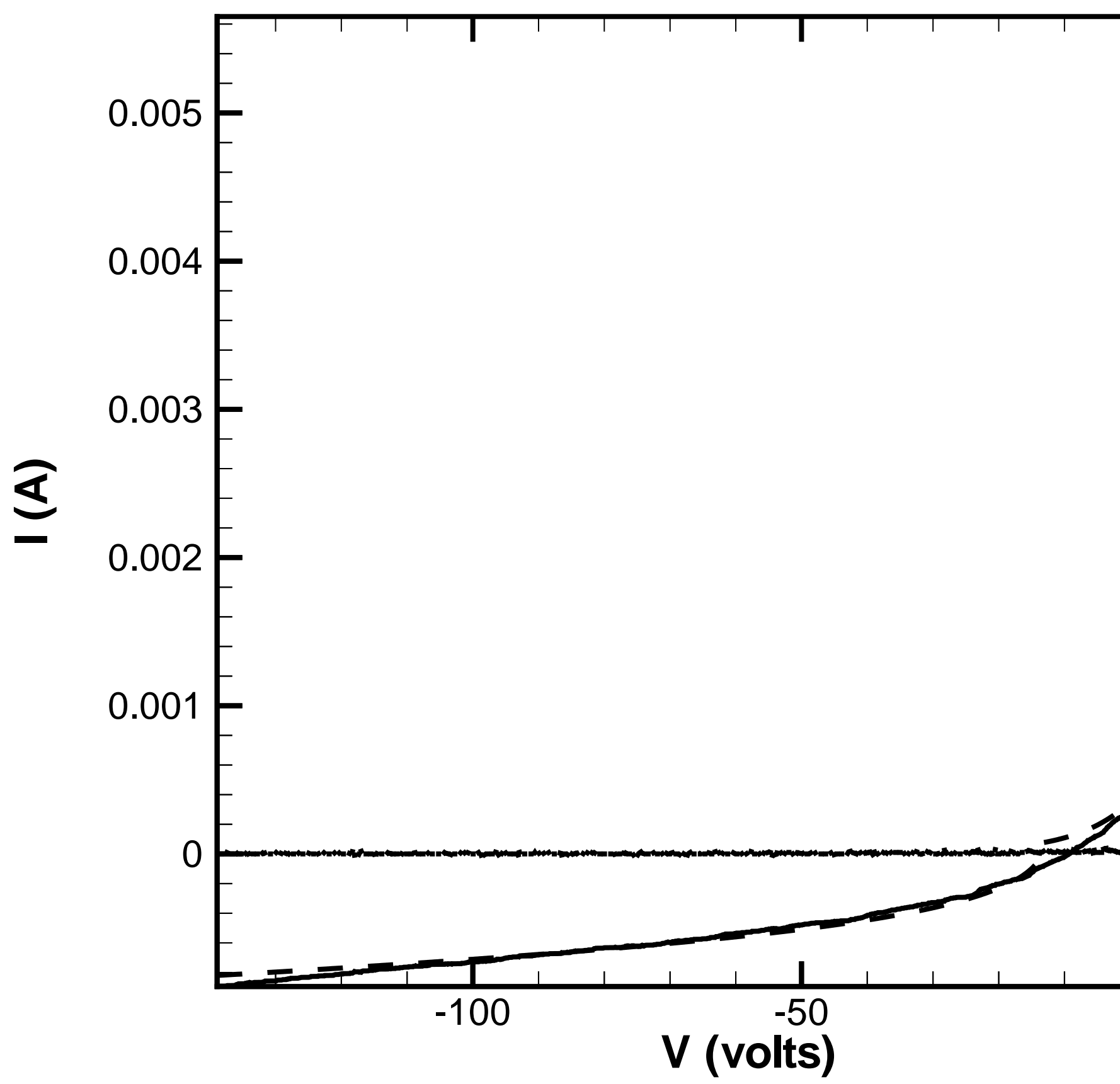




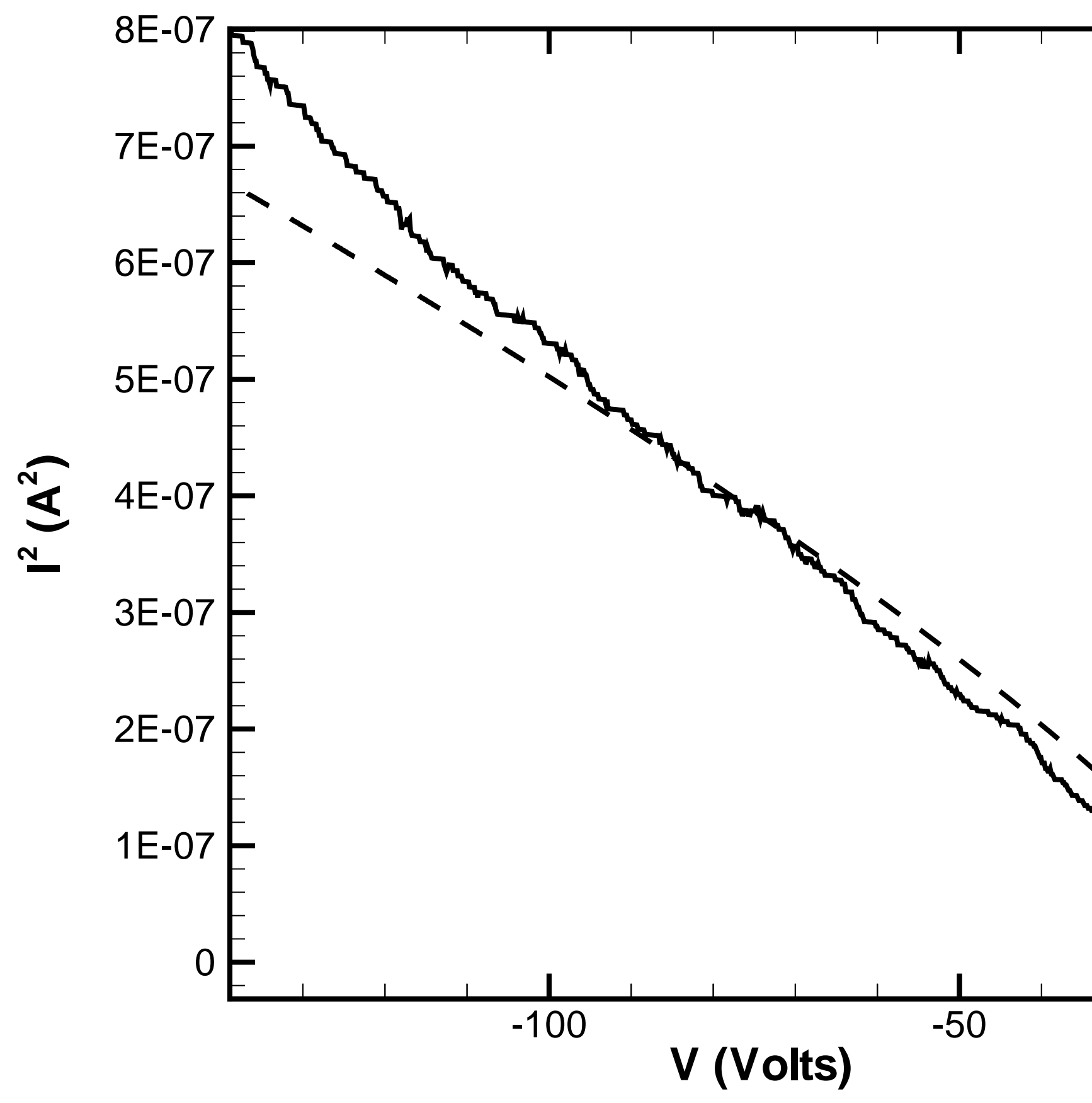




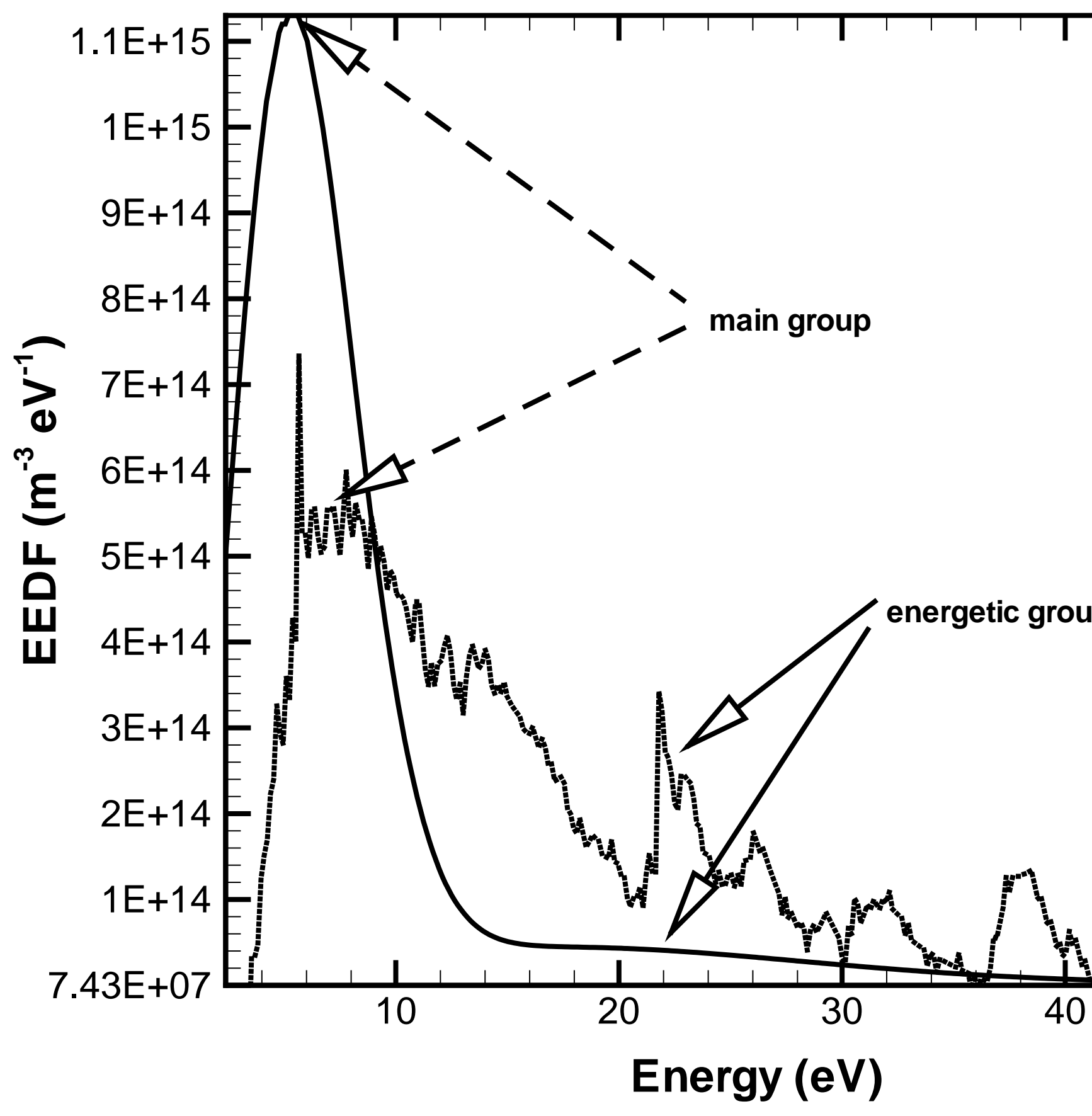

